\title{
Acute exacerbation of COPD is associated with fourfold elevation of cardiac troponin $T$
}

\author{
Vidar Søyseth, ${ }^{1}$ Rahul Bhatnagar, ${ }^{1}$ Nils Henrik Holmedahl, ${ }^{2}$ Anke Neukamm, ${ }^{1}$ \\ Arne Didrik Høiseth, ${ }^{1}$ Tor-Arne Hagve, ${ }^{1}$ Gunnar Einvik, ${ }^{1}$ Torbjørn Omland ${ }^{1}$
}

\begin{abstract}
${ }^{1}$ Department of Medicine, Akershus University Hospital, University of Oslo, Lørenskog, Norway and Faculty of Medicine, University of Oslo, Campus Akershus University Hospital, Lørenskog ${ }^{2}$ Glittreklinikken, Hakadal, Norway
\end{abstract}

\section{Correspondence to}

Dr Vidar Søyseth, Department of Medicine, Akershus University Hospital, University of Oslo, Sykehusvn 25, Lørenskog 1478, Norway; vidar.soyseth@medisin.uio.no

Received 4 July 2012 Revised 21 August 2012 Accepted 22 August 2012 Published Online First 28 September 2012

\section{Sinked}

- http://dx.doi.org/10.1136/ heartjnl-2012-302969

To cite: Søyseth $\mathrm{V}$ Bhatnagar $\mathrm{R}$,

Holmedahl $\mathrm{NH}$, et al. Heart 2013, 99, 122-126.

\begin{abstract}
Objective To investigate if acute exacerbation of chronic obstructive pulmonary disease (AECOPD) is associated with myocardial injury, expressed as elevated high sensitive cardiac troponin T (hs-cTnT), and to identify determinants of hs-cTnT in chronic obstructive pulmonary disease (COPD) patients.

Design In a cross-sectional study, hs-cTnT in patients hospitalised for AECOPD was compared with hs-cTnT in COPD patients in their stable state.
\end{abstract}

Setting The study was conducted at a teaching and a pulmonary rehabilitation clinic.

Participants Consecutive admissions to participating units for the years 2010-2011 meeting objective, standardised criteria for AECOPD and stable COPD.

Main outcomes Ratio of hs-cTnT in hospitalised AECOPD patients compared with stable COPD patients. Change in the ratio of hs-cTnT per unit increase of relevant covariables.

Results The geometric mean of hs-cTnT in the index group was $25.8 \mathrm{ng} / \mathrm{l}(95 \% \mathrm{Cl} 21.1$ to 31.7$)$ compared with $4.55 \mathrm{ng} / \mathrm{l}(95 \% \mathrm{Cl} 3.72$ to 5.67$)$ in the reference group. After inclusion of relevant covariables, multiple linear regression analyses showed that the ratio between hs-cTnT in AECOPD patients and the references was $4.26(95 \% \mathrm{Cl} 3.02$ to 6.00$)$ and that hs-cTnT increased 1.41-fold (95\% Cl 1.20 to 1.68), for each quartile increase in leucocyte count in stable COPD but not in AECOPD. Higher hs-cTnT levels were also associated with the presence of pathological $q$-waves $(p=0.012)$ and electrocardiographic left ventricular hypertrophy $(p=0.039)$, long-term oxygen treatment $(p=0.002)$ and decreasing forced expiratory volume in $1 \mathrm{~s}(\mathrm{p}=0.014)$. A significant univariable association between cTnT and arterial hypoxaemia was also found but this association was attenuated almost to a zero effect after inclusion of relevant covariates.

Conclusions AECOPD is associated with higher hscTnT as compared with stable COPD. In stable COPD hs-cTnT appears to be positively associated with indices of COPD severity, whereas we were unable to identify significant determinants of hs-cTnT in AECOPD.

Chronic obstructive pulmonary disease (COPD) is characterised by airflow limitation that is not reversible and is usually progressive. ${ }^{1}$ In the majority of the patients, the disease is accompanied with chronic respiratory symptoms, foremost dyspnoea, cough and phlegm. A large proportion of the patients have considerable comorbidity that frequently has significant consequences for the morbidity as well as the mortality of the patients. ${ }^{2}$ These comorbidities include osteoporosis, lung cancer and cardiovascular diseases, among others. Some patients experience periods with worsening of symptoms, foremost increased dyspnoea, that are frequently accompanied with acute respiratory failure needing treatment in hospital. Such periods are called acute exacerbation of COPD (AECOPD). ${ }^{3} 4$

It has been found that a considerable number of patients who are hospitalised for AECOPD have elevated cardiac troponin. ${ }^{5-7}$ Troponin is a protein that is bound to the actin filaments of myocytes. ${ }^{8} 9$ Troponin can be divided into three subclasses: troponin C, I and T. Troponin I and T that are specific for the cardiac myocytes and are often referred to as cardiac troponin, whereas troponin $\mathrm{C}$ is found in skeletal muscles. Thus, elevated levels of cardiac troponin $\mathrm{T}(\mathrm{cTnT})$ or cardiac troponin I in peripheral blood are regarded as a marker of myocardial injury, including myocardial infarction. However, elevated cTnT (or cardiac troponin I) has been observed in several other settings than acute myocardial infarction, such as septicaemia and pulmonary embolism. ${ }^{10-13}$ Usually, such elevated levels of troponin are associated with increased mortality.

In patients hospitalised for AECOPD, elevated levels of troponin are associated with increased mortality. $^{5} 614{ }^{15}$ It has been found that the level of cTnT in AECOPD is associated with increased heart rate and creatinine. ${ }^{16}{ }^{17}$ It is, however, not known if the troponin level is associated with AECOPD or any other index of the severity of the disease. Hence, in the current study we have compared cTnT in patients hospitalised for AECOPD with cTnT in COPD patients in their stable state. In both groups, we had spirometry results during stable periods.

The objective of the study was twofold. We wanted to investigate: (1) if cTnT was higher with AECOPD than stable COPD and (2) the association between $\mathrm{cTnT}$ and indices of COPD severity.

\section{MATERIALS AND METHODS}

All the patients had COPD confirmed by spirometry in their stable state within the last 5 years. If reversibility test was not available, the diagnosis was clinically confirmed by a chest physician. Moreover, all the patients were between 40 and 79 years old and they should have a cumulative tobacco consumption of 10 pack-years or more (1 pack-year: 20 cigarettes daily for 1 year). Both current smokers and former smokers were included. They completed a questionnaire about respiratory symptoms, comorbid conditions and smoking history. In addition, they also completed a COPD Assessment Test questionnaire. ${ }^{18}$ 
They gave written consent, and the study was approved by the Regional Ethics Committee.

\section{INDEX GROUP (AECOPD, N=50)}

The index group consisted of patients hospitalised for AECOPD at the Akershus University Hospital from February 2010 to December 2011. They should have performed spirometry at the hospital out-clinic within the last 5 years before admittance (median, 10 and 90 percentiles: 8, 3 and 36 months). Blood samples (haemoglobin, peripheral leucocytes, creatinine and cTnT), arterial blood gases and blood pressure were measured on admittance in the emergency room. An ECG was recorded simultaneously. Heart rate was obtained from the ECG. Patients were enrolled in the study on the 3 rd to 5 th day after admittance. At the day of inclusion, patients were weighted and completed the questionnaires. Characteristics of the index group are shown in table 1 .
REFERENCES (STABLE COPD, $\mathrm{N}=124$ )

The references were recruited at a lung rehabilitation hospital (the Glittre Clinic). The day after admittance to the hospital, peripheral venous blood and arterial blood gases were sampled. Pulmonary function tests were also carried out on the second day and they completed the questionnaires.

\section{MEASUREMENTS}

Spirometry was performed as recommended by European Respiratory Society (ERS)/American Thoracic Society (ATS) using the European Community for Coal and Steel (ECCS) reference equations. ${ }^{19}{ }^{20} \mathrm{C}$ reactive protein (CRP) was analysed on a Vitros 5.1 FS instrument (Ortho Clinical Diagnostics Rochester, NY, USA) using reagents from Sentinel Diagnostics, Milan, Italy (AECOPD). High sensitive CRP (hs-CRP) and TnT were analysed on a Cobas e602 instrument (Roche Diagnostics, Mannheim, Germany) with reagents also from

Table 1 Characteristics of the total cohort, COPD patients at hospitalisation for exacerbation (AECOPD) and in stable state

\begin{tabular}{|c|c|c|c|c|}
\hline Covariate & Total & $\operatorname{AECOPD}(n=50)$ & Stable state $(n=124)$ & $\mathrm{p}$ Value \\
\hline \multicolumn{5}{|l|}{ Demographic data } \\
\hline Age, years mean (SD) & $65.0(7.9)$ & $66.5(6.8)$ & $64.3(8.2)$ & 0.093 \\
\hline Female, n (\%) & $92(53)$ & $25(50)$ & $67(54)$ & 0.630 \\
\hline $\mathrm{BMI}, \mathrm{kg} / \mathrm{m}^{2}$ mean $(\mathrm{SD})$ & $24.2(5.5)$ & $23.2(5.4)$ & $24.6(5.4)$ & 0.086 \\
\hline \multicolumn{5}{|l|}{ Smoking habit } \\
\hline Pack-years, mean (SD) & $35.7(21.1)$ & $42.6(19.2)$ & $33.0(21.3)$ & 0.006 \\
\hline Current smoker, n (\%) & $39(22)$ & $8(16)$ & $31(25)$ & 0.198 \\
\hline \multicolumn{5}{|l|}{ Clinical data } \\
\hline Systolic BP, mm Hg mean (SD) & $138(22)$ & $138(28)$ & $138(19)$ & 0.845 \\
\hline $\mathrm{HR}, 1 /$ min mean $(\mathrm{SD})$ & $85(22)$ & $106(22)$ & $76(15)$ & $<0.001$ \\
\hline Arterial HT, n (\%) & $65(37)$ & $14(28)$ & $51(41)$ & 0.105 \\
\hline$C A D, n(\%)$ & $28(16)$ & $12(24)$ & $16(13)$ & 0.071 \\
\hline CAT score, mean (SD) & $21.1(7.6)$ & $28.0(5.7)$ & $18.1(6.2)$ & $<0.001$ \\
\hline Long-term $\mathrm{O}_{2}$ treatment, $\mathrm{n}(\%)$ & $21(12)$ & 7 (14) & $14(11)$ & 0.633 \\
\hline \multicolumn{5}{|l|}{ ECG, n (\%) } \\
\hline Atrial fibrillation & $7(4)$ & $6(13)$ & $1(1)$ & 0.001 \\
\hline q-Wave & $12(7)$ & $1(2)$ & $11(9)$ & 0.115 \\
\hline T-wave inversion & $20(12)$ & $6(12)$ & $14(12)$ & 0.939 \\
\hline Left bundle branch block & $4(2)$ & $1(2)$ & $3(2)$ & 0.905 \\
\hline Left ventricular hypertrophy & $5(3)$ & $1(2)$ & $4(3)$ & 0.684 \\
\hline \multicolumn{5}{|l|}{ Spirometry } \\
\hline FVC, L mean (SD) & $2.46(0.93)$ & $2.11(0.68)$ & $2.60(0.98)$ & 0.002 \\
\hline $\mathrm{FEV}_{1}, \mathrm{~L}$ mean $(\mathrm{SD})$ & $1.07(0.51)$ & $0.85(0.41)$ & $1.15(0.53)$ & $<0.001$ \\
\hline $\mathrm{FEV}_{1}$ to $\mathrm{FVC}$, mean (SD) & $0.45(0.15)$ & $0.40(0.12)$ & $0.47(0.16)$ & 0.009 \\
\hline \multicolumn{5}{|l|}{ Arterial blood gases, kPa mean (SD) } \\
\hline $\mathrm{P}_{\mathrm{O}_{2}}$ & $8.85(1.51)$ & $8.16(1.82)$ & $9.16(1.24)$ & $<0.001$ \\
\hline $\mathrm{P}_{\mathrm{CO}_{2}}$ & $5.89(1.59)$ & $6.55(2.38)$ & $5.60(0.95)$ & $<0.001$ \\
\hline \multicolumn{5}{|l|}{ Laboratory data } \\
\hline Haemoglobin, g/dl mean (SD) & $14.3(1.4)$ & $13.7(1.4)$ & $14.5(1.4)$ & $<0.001$ \\
\hline Leucocytes, $10^{-9} / /$ mean (SD) & $9.2(3.7)$ & $12.9(4.5)$ & $7.9(2.1)$ & $<0.001$ \\
\hline Creatinine, $\mu \mathrm{mol} / / \mathrm{l}$ mean (SD) & $75.9(20.7)$ & $69.3(21.1)$ & $75.9(20.3)$ & 0.064 \\
\hline CRP, mg/l geometric mean & 6.26 & 38.30 & 2.75 & $<0.001$ \\
\hline IL-6, pg/ml geometric mean & - & - & 2.34 & - \\
\hline hs-cTnT, ng/l n (\%) & & & & $<0.001$ \\
\hline$<14.0$ & $98(61)$ & $9(18)$ & $89(81)$ & \\
\hline $14.0-39.9$ & $41(26)$ & $26(52)$ & $15(14)$ & \\
\hline$\geq 40.0$ & $21(13)$ & $15(30)$ & $6(5)$ & \\
\hline
\end{tabular}

All spirometry data were measured in stable state.

AECOPD, acute exacerbation of chronic obstructive pulmonary disease; BMI, body mass index; BP, blood pressure; CAD, coronary artery disease; CAT, COPD Assessment Test; COPD,

chronic obstructive pulmonary disease; CRP, C reactive protein; FEV 1 , forced expiratory volume in $1 \mathrm{~s}$; FVC, forced vital capacity; HR, heart rate; hs-cTnT, high sensitive cardiac troponin $\mathrm{T}$; HT, hypertension; IL, interleukin; one pack-year: 20 cigarettes daily during 1 year. 
Roche (stable COPD). This is a high sensitive method for cTnT (hs-cTnT); that is, the detection limit is $3.0 \mathrm{ng} / \mathrm{l}$. In the reference group, 99th percentile was $14 \mathrm{ng} / \mathrm{l}$ in accordance with others. ${ }^{9}$ The lowest level with $10 \%$ coefficient of variation was $13 \mathrm{ng} / \mathrm{l}$.

\section{STATISTICAL ANALYSES}

The statistical analyses were performed in three steps. First, we investigated the association between COPD and the relevant covariables. In these analyses, we used Student $t$ test to investigate the univariable difference between continuous variables in the index group and the reference group and $\chi^{2}$ test to compare univariable relationships between categorical variables in each group. Second, we investigated the univariable association between hs-cTnT and relevant covariables. In these analyses, the dependent variable was expressed as log-transformed hs-cTnT $(\ln ($ hs-cTnT)) using ordinary least square regression (continuous covariables) and Student $\mathrm{t}$ test for dichotomous covariables. Continuous explanatory variables were categorised in quartiles. Pearson's correlation analyses were also performed in order to investigate the association between relevant continuous determinants of hs-cTnT.

Third, covariables that were associated with COPD or hs-cTnT with a corresponding $\mathrm{p}$ value $<0.2$ were included in a multiple linear regression model using the log-transformation of hs-cTnT as the dependent variable. The following variables were included in these analyses as explanatory variables in addition to AECOPD: gender, age, body mass index, smoking habits (ie, current smoking (yes/no) and cumulative tobacco consumption (pack-years)), heart rate, history of arterial hypertension or coronary artery disease (ie, previous myocardial infarction, cardiac artery bypass surgery or percutaneous coronary intervention), long-term oxygen treatment, ECG features (ie, atrial fibrillation, Q-wave T-wave inversion, left bundle branch block), spirometry (ie, forced vital capacity (FVC), forced expiratory volume in $1 \mathrm{~s}$ $\left(\mathrm{FEV}_{1}\right), \mathrm{FEV}_{1}$ to $\mathrm{FVC}$ ratio), arterial blood gases $\left(\mathrm{O}_{2}\right.$ and $\left.\mathrm{CO}_{2}\right)$, haemoglobin, blood leucocytes, CRP (in stable COPD: hs-CRP), creatinine and AECOPD (yes vs no). If hs-cTnT was lower than the detection limit it was replaced by $1.5 \mathrm{ng} / \mathrm{l}(50 \%$ of the detection limit). Symptoms were recorded using a validated questionnaire. $^{18}$

Finally, the model was reduced by backward elimination as follows: the covariables that were associated with $\ln ($ hs-cTnT) with a $\mathrm{p}$ value $<0.1$ were removed from the model one by one provided that the association between $\ln (\mathrm{hs}-\mathrm{cTnT})$ and AECOPD did change $<20 \%$ after the removal of each covariable. Gender and age were withheld in the model regardless of the corresponding $\mathrm{p}$ value. The coefficients in the final model were antilogged. These antilogged coefficients can be interpreted as the ratio between hs-cTnT and one unit increase of the corresponding covariable.

The analyses were performed using the statistical software Stata V.11.2 (http://www.stata.com).

\section{RESULTS}

The geometric mean of hs-cTnT and the corresponding 95\% CI (in parentheses) in the index group and the references were 25.8 (21.1 to 31.7 ) $\mathrm{ng} / \mathrm{l}$ and 4.55 (3.72 to 5.67 ) ng/l, respectively. The crude ratio of hs-cTnT between the AECOPD patients and the stable COPD patients was 5.67 (4.09 to 7.86). A doseresponse relationship between hs-cTnT and $\mathrm{FEV}_{1}$ as per cent of predicted was also indicated (figure 1). Results of the univariable analyses between hs-cTnT and several variables are shown in table 2A,B. Although only the spirometry variables had a

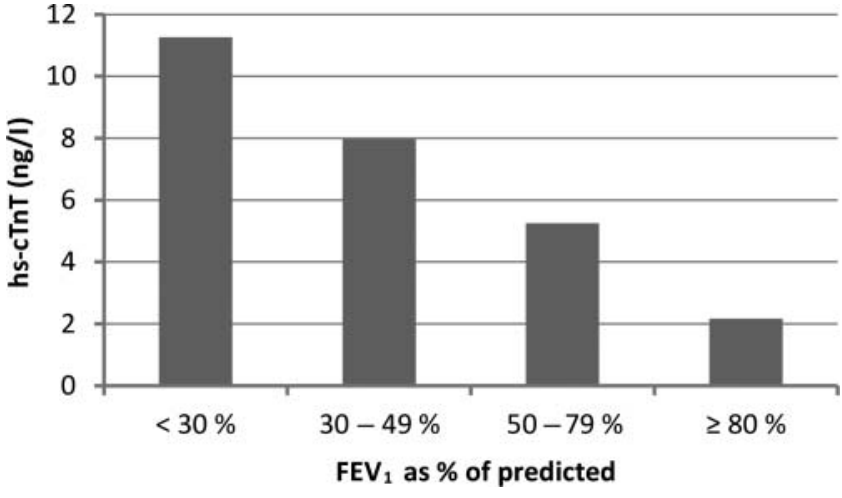

Figure 1 Geometric mean of highly sensitive troponin T (hs-cTnT) by forced expiratory volume in $1 \mathrm{~s}\left(\mathrm{FEV}_{1}\right)$ as $\%$ of predicted.

correlation coefficient larger than 0.60 , several of the covariables were mutually confounded. Consequently, many of the covariables that showed a highly significant univariate association with hs-cTnT turned out to be non-significant in the multivariable regression model. Except from the spirometry results, the correlations between the covariables were moderate and did not cause cumbersome challenges in the multivariate analyses. Thus, the final model should be considered robust.

The results of the multivariable analyses are shown in table 3 . In the total cohort, hs-cTnT was lower in female subjects compared with male subjects $(\mathrm{p}=0.068$; ie, a trend towards borderline significance). Moreover, hs-cTnT was significantly higher in patients having q-waves or left ventricular hypertrophy in their ECG as well as in patients with long-term oxygen treatment than their counterparts. A significant increase in hs-cTnT with decreasing $\mathrm{FEV}_{1}$ was found. The association between creatinine and hs-cTnT was of borderline significance in the total cohort but significant in stable COPD patients $(p=0.028)$. Foremost, it was 4.26-fold higher in AECOPD patients than the references (table 3). Interestingly, we found a highly significant increase in hs-cTnT with increasing peripheral leucocyte count $(\mathrm{p}<0.001)$ in the references but not in AECOPD group $(p=0.585)$. Briefly, in the index group we did not find any significant association between hs-cTnT and the covariables in the final model. Neither

Table 2A Geometric mean of high sensitive cardiac troponin T (hs-cTnT in $\mathrm{ng} / \mathrm{l}$ ) by dichotomous covariates and the ratio of geometric mean of hs-cTnT

\begin{tabular}{|c|c|c|c|c|}
\hline \multirow[b]{2}{*}{ Covariate } & \multicolumn{2}{|c|}{ Covariate present } & \multirow[b]{2}{*}{ Ratio } & \multirow[b]{2}{*}{ p Value } \\
\hline & Yes & No & & \\
\hline \multicolumn{5}{|l|}{ Demographic data } \\
\hline Female & 6.27 & 10.21 & 0.61 & 0.014 \\
\hline Current smoker & 5.06 & 8.61 & 0.59 & 0.031 \\
\hline \multicolumn{5}{|l|}{ Clinical data } \\
\hline ECG, q-wave & 10.83 & 7.64 & 1.42 & 0.360 \\
\hline ECG, LVH & 16.88 & 7.65 & 2.21 & 0.170 \\
\hline ECG, T-wave inv. & 11.85 & 7.39 & 1.60 & 0.119 \\
\hline ECG, atrial fibrillation & 20.7 & 7.50 & 2.76 & 0.037 \\
\hline Arterial HT & 8.44 & 6.65 & 1.27 & 0.250 \\
\hline$C A D$ & 8.63 & 7.54 & 1.14 & 0.609 \\
\hline Long-term $\mathrm{O}_{2}$ treatment & 14.11 & 7.21 & 1.95 & 0.026 \\
\hline
\end{tabular}


Table 2B Geometric mean of high sensitive cardiac troponin T (hs-cTnT) by quartiles $(\mathrm{Qn})$ and the ratio of hs-cTnT between the Qn of the covariates listed in column 1

\begin{tabular}{|c|c|c|c|c|c|c|}
\hline \multirow[b]{2}{*}{ Covariate } & \multicolumn{4}{|c|}{ Quartile (Qn) of covariate } & \multirow[b]{2}{*}{ Ratio } & \multirow[b]{2}{*}{$p$ Value } \\
\hline & Q1 & Q2 & Q3 & Q4 & & \\
\hline \multicolumn{7}{|l|}{ Demographic data } \\
\hline Age $(59 ; 64 ; 68)$ years & 4.40 & 10.0 & 5.75 & 13.3 & 1.32 & 0.002 \\
\hline BMI $(20 ; 24 ; 27) \mathrm{kg} / \mathrm{m}^{2}$ & 9.68 & 6.56 & 10.61 & 5.45 & 0.88 & 0.136 \\
\hline Pack-years & 5.21 & 6.64 & 10.40 & 9.55 & 1.25 & 0.011 \\
\hline \multicolumn{7}{|l|}{ Clinical data } \\
\hline Heart rate $(67 ; 81 ; 96) 1 / \mathrm{min}$ & 3.80 & 4.89 & 8.69 & 19.79 & 1.75 & $<0.001$ \\
\hline $\begin{array}{l}\text { Systolic BP } \\
(123 ; 136 ; 150) \mathrm{mm} \mathrm{Hg}\end{array}$ & 8.47 & 9.03 & 10.07 & 5.67 & 0.87 & 0.123 \\
\hline CAT score $(16 ; 20 ; 27)$ & 3.19 & 4.66 & 10.84 & 15.46 & 1.74 & $<0.001$ \\
\hline \multicolumn{7}{|l|}{ Spirometry } \\
\hline FVC $(1.78 ; 2.31 ; 3.07)$ I & 11.0 & 8.03 & 8.28 & 4.66 & 0.77 & 0.005 \\
\hline $\mathrm{FEV}_{1}(0.66 ; 0.89 ; 1.38) \mathrm{I}$ & 11.0 & 9.80 & 6.23 & 4.40 & 0.76 & 0.003 \\
\hline $\mathrm{FEV}_{1}$ to $\mathrm{FVC}(0.34 ; 0.42 ; 0.51)$ & 8.94 & 9.59 & 6.91 & 5.91 & 0.85 & 0.085 \\
\hline \multicolumn{7}{|l|}{ Arterial blood gases and $\mathrm{pH}$} \\
\hline $\mathrm{P}_{\mathrm{O}_{2}}(8.1 ; 9.0 ; 9.8) \mathrm{kPa}$ & 12.0 & 7.8 & 7.2 & 4.8 & 0.75 & 0.003 \\
\hline $\mathrm{P}_{\mathrm{CO}_{2}}(5.0 ; 5.6 ; 6.3) \mathrm{kPa}$ & 5.7 & 6.6 & 6.8 & 18.6 & 1.39 & 0.001 \\
\hline pH (7.39; 7.42; 7.44) & 10.2 & 6.3 & 6.3 & 8.7 & 0.94 & 0.489 \\
\hline \multicolumn{7}{|l|}{ Laboratory data } \\
\hline $\begin{array}{l}\text { Haemoglobin } \\
(13.3 ; 14.4 ; 15.2) \mathrm{g} / \mathrm{dl}\end{array}$ & 11.3 & 8.53 & 4.16 & 8.71 & 0.85 & 0.094 \\
\hline $\begin{array}{l}\text { Leucocytes } \\
(6.9 ; 8.1 ; 11.1) 10^{-9} / /\end{array}$ & 4.39 & 3.45 & 8.13 & 23.01 & 1.82 & $<0.001$ \\
\hline $\operatorname{CRP}(1.9 ; 4.5 ; 23.5) \mathrm{mg} / \mathrm{l}$ & 3.75 & 3.98 & 4.93 & 5.40 & 0.139 & 0.164 \\
\hline Creatinine $(57 ; 71 ; 85) \mu \mathrm{mol} / \mathrm{l}$ & 9.08 & 6.39 & 6.09 & 10.28 & 1.04 & 0.680 \\
\hline
\end{tabular}

hypoxaemia nor history of coronary heart disease was significantly associated with hs-cTnT. Also, the association between hs-cTnT and chest pain was non-significant. There was no association between hs-CRP and hs-cTnT in stable COPD patients or CRP and hs-cTnT in AECOPD even after replacing leucocytes with hs-CRP/CRP in the multivariate analyses. Moreover, the relationship between IL-6 and hs-cTnT in stable COPD was non-significant $(p=0.290)$. The final model resulted in
R-squared adjusted of $58.0 \%$, that is, a fairly good fit to the data. F-statistic for heteroskedasticity revealed $\mathrm{p}=0.298$.

\section{DISCUSSION}

In this study, we have found that AECOPD is associated with significant elevation of troponin $\mathrm{T}$ measured by a highly sensitive assay. Moreover, we found that in stable COPD hs-cTnT increased with decreasing pulmonary function $\left(\mathrm{FEV}_{1}\right)$, increasing peripheral blood leucocyte count and serum creatinine. Long-term oxygen treatment and left ventricular hypertrophy were also associated with significantly elevated hs-cTnT. Among the AECOPD patients we did not find similar associations between hs-cTnT and any of these covariables.

These results may have several explanations, such as acute coronary syndrome, pulmonary embolism or acute heart failure, as all these disorders have symptoms that can mimic AECOPD.

However, we found no association between retrosternal chest pains and hs-cTnT and the prevalence of T-wave inversion did not differ between the groups. Furthermore, none of the patients were diagnosed with myocardial infarction at discharge from the hospital. Nonetheless, non-ST elevation myocardial infarction cannot be excluded as undiagnosed subendocardial infarction has been found in autopsies of patients who have died in hospital during AECOPD. ${ }^{21}$ Thus, the possibility of atherosclerotic myocardial infarction in COPD patients hospitalised for worsening of symptoms warrants further investigation. As we found a highly significant positive association between hs-cTnT and the prevalence of q-waves as well left ventricular hypertrophy, the results from echocardiography and MRI would be desirable.

The prevalence of pulmonary embolism and congestive heart failure is also elevated in COPD patients and the symptoms of these disorders may mimic AECOPD. ${ }^{22}{ }^{23}$ None of the patients in the index group were diagnosed with pulmonary embolism or acute congestive heart failure.

Type 2 myocardial infarction due to hypoxaemia should also be considered. ${ }^{24}$ Actually, we found a strong positive univariable relationship between hs-cTnT and arterial oxygen tension. Nonetheless, this association was markedly attenuated after inclusion of AECOPD to the model. We have previously failed to find any association between troponin $\mathrm{T}$ and arterial hypoxaemia in AECOPD patients at admittance to the hospital. ${ }^{25} 26$ Admittedly, the lack of association between hypoxaemia and elevated hs-cTnT could be confounded by AECOPD. Thirty of the patients among the references had arterial $\mathrm{O}_{2}$ tension less than

Table 3 Relative change in hs-cTnT (hs-cTnT ${ }_{x+\Delta x} / h_{-1}-\operatorname{TnT}_{x}(95 \% \mathrm{Cl}$ in parentheses) by unit change $(\Delta \mathrm{x})$ ) of the covariables

\begin{tabular}{|c|c|c|c|c|}
\hline \multirow[b]{2}{*}{ Covariate } & \multirow[b]{2}{*}{ Change of covariable $(\Delta x)$} & \multicolumn{3}{|l|}{ Hs-cTnT ${ }_{x+\Delta x} /$ hs-cTnT ${ }_{x}$} \\
\hline & & Total cohort & Exacerbation & Stable state \\
\hline Gender & Female versus male & $0.74(0.54$ to 1.02$)$ & 0.85 (0.53 to 1.35$)$ & 0.69 (0.44 to 1.05$)$ \\
\hline Age & Quartile & $1.05(0.92$ to 1.20$)$ & $1.03(0.83$ to 1.28$)$ & $1.03(0.87$ to 1.21$)$ \\
\hline LTOT & Yes versus no & 1.94 (1.27 to 2.98$)$ & $1.20(0.62$ to 2.31$)$ & 2.66 (1.57 to 4.50$)$ \\
\hline ECG: q-wave & Yes versus no & 2.00 (1.17 to 3.44$)$ & NA & 1.89 (1.08 to 3.28$)$ \\
\hline ECG: LVH & Yes versus no & 2.33 (1.04 to 5.20$)$ & $0.89(0.20$ to 4.06$)$ & 3.36 (1.32 to 8.56$)$ \\
\hline $\mathrm{FEV}_{1}$ & Quartile & 0.81 (0.69 to 0.96$)$ & $1.03(0.78$ to 1.32$)$ & 0.73 (0.60 to 0.89$)$ \\
\hline Leucocytes & Quartile & 1.30 (1.14 to 1.50$)$ & $1.06(0.83$ to 1.36$)$ & 1.41 (1.20 to 1.68$)$ \\
\hline Creatinine & Quartile & $1.15(1.00$ to 1.33$)$ & $0.92(0.74$ to 1.15$)$ & 1.21 (1.01 to 1.47$)$ \\
\hline AECOPD & Yes versus no & $4.26(3.02$ to 6.00$)$ & - & - \\
\hline
\end{tabular}

AECOPD, acute exacerbation of chronic obstructive pulmonary disease; FEV $\mathrm{NA}$, not available (only one patient). 
$8.0 \mathrm{kPa}$. Thus, it is unlikely that the imbalance between the groups regarding oxygen tension should confound the relationship between hs-cTnT and arterial oxygen tension noteworthy. However, this observation could have several pitfalls, such as oxygen supplementation during transportation to the hospital. Nonetheless, it is remarkable that any real association should be distorted to the null effect as nearly $50 \%$ of the AECOPD patients had arterial $\mathrm{P}_{\mathrm{CO}_{2}}<8.0 \mathrm{kPa}$ on admittance to the hospital.

In stable COPD, we found a strong and consistent association between elevated hs-cTnT and leucocyte count, long-term oxygen treatment and decreasing $\mathrm{FEV}_{1}$; that is, indices of systemic inflammation and COPD severity. It appears that this effect is not mediated by CRP or IL- 6 as the multivariate analyses did not show any evidence of an association between these two inflammatory markers and hs-cTnT even if we removed leucocytes from the model. In the stable state, there is a strong relationship between inflammatory markers in sputum and peripheral blood as well as pulmonary function tests. ${ }^{27}$ In AECOPD this association is, however, attenuated. ${ }^{28} 29$

The main limitation of this study is the cross-sectional design. Hence, none of relationships between hs-cTnT and the explanatory variables can be interpreted causally. Moreover, the small sample size, for example, the results could be attributed to sampling error. This explanation is, however, unlikely as the distribution variables like age, gender and smoking habits are fairly comparable with other studies of AECOPD. 71527

\section{CONCLUSIONS}

Troponin Twas significantly elevated in COPD patients hospitalised for exacerbation compared with stable COPD. In the stable state, elevated troponin T was associated with increasing leucocyte count and decreasing FEV1. The determinants for troponin elevation during AECOPD remain, however, unknown.

\section{Acknowledgements The authors thank Ms. Sausann Christin Brunell for} laboratory work and Mrs Vigdis Bakkelund for handling of the blood samples. Ethics approval was provided by the Regional Committee for medical and Health Ethics South East, Norway.

Disclaimer None.

Contributors VS: idea, design, data collection and analyses, manuscript preparation. RB and NHH: data collection and manuscript preparation. AN and ADH: data analyses and manuscript preparation. T-AH: chemical analyses and manuscript preparation. TO: design and manuscript preparation. GE: design, data analyses and manuscript preparation.

Funding None.

Competing interest None.

Patient consent Obtained.

Provenance and peer review Not commissioned; externally peer reviewed.

\section{REFERENCES}

1 Rabe KF, Hurd S, Anzueto A, et al. Global strategy for the diagnosis, management, and prevention of chronic obstructive pulmonary disease: GOLD executive summary. Am J Respir Crit Care Med 2007;176:532-55.

2 Barnes PJ, Celli BR. Systemic manifestations and comorbidities of COPD. Eur Respir J 2009;33:1165-85.

3 Donaldson GC, Wedzicha JA. COPD exacerbations 0.1: epidemiology. Thorax 2006;61:164-8.
4 Wedzicha JA, Seemungal TA. COPD exacerbations: defining their cause and prevention. Lancet 2007;370:786-96.

5 Baillard C, Boussarsar M, Fosse JP, et al. Cardiac troponin I in patients with severe exacerbation of chronic obstructive pulmonary disease. Intensive Care Med 2003;29:584-9.

6 Brekke PH, Omland T, Holmedal SH, et al. Troponin T elevation and long-term mortality after chronic obstructive pulmonary disease exacerbation. Eur Respir J 2008:31:563-70.

7 Harvey MG, Hancox RJ. Elevation of cardiac troponins in exacerbation of chronic obstructive pulmonary disease. Emerg Med Australas 2004;16:212-15.

8 Babuin L, Jaffe AS. Troponin: the biomarker of choice for the detection of cardiac injury. CMAJ 2005;173:1191-202.

9 Omland T. New features of troponin testing in different clinical settings. J Intern Med 2010;268:207-17.

10 Chang CL, Robinson SC, Mills GD, et al. Biochemical markers of cardiac dysfunction predict mortality in acute exacerbations of COPD. Thorax 2011;66:764-8.

11 Favory R, Neviere R. Significance and interpretation of elevated troponin in septic patients. Crit Care 2006;10:224.

12 Hoiseth AD, Neukamm A, Karlsson BD, et al. Elevated high-sensitivity cardiac troponin $T$ is associated with increased mortality after acute exacerbation of chronic obstructive pulmonary disease. Thorax 2011:66:775-81.

13 Jeremias A, Gibson CM. Narrative review: alternative causes for elevated cardiac troponin levels when acute coronary syndromes are excluded. Ann Intern Med 2005;142:786-91.

14 Lankeit M, Friesen D, Aschoff J, et al. Highly sensitive troponin T assay in normotensive patients with acute pulmonary embolism. Eur Heart $J$ 2010;31:1836-44.

15 Rosjo $H$, Varpula M, Hagve TA, et al. Circulating high sensitivity troponin T in severe sepsis and septic shock: distribution, associated factors, and relation to outcome. Intensive Care Med 2011:37:77-85.

16 Abbas NA, John RI, Webb MC, et al. Cardiac troponins and renal function in nondialysis patients with chronic kidney disease. Clin Chem 2005:51:2059-66

17 Freda BJ, Tang WH, Van LF, et al. Cardiac troponins in renal insufficiency: review and clinical implications. J Am Coll Cardiol 2002:40:2065-71.

18 Jones PW, Harding G, Berry P, et al. Development and first validation of the COPD Assessment Test. Eur Respir J 2009:34:648-54.

19 Miller MR, Hankinson J, Brusasco V, et al. Standardisation of spirometry. Eur Respir J 2005;26:319-38.

20 Quanjer PH, Tammeling GJ, Cotes JE, et al. Lung volumes and forced ventilatory flows. Report Working Party Standardization of Lung Function Tests, European Community for Steel and Coal. Official Statement of the European Respiratory Society. Eur Respir J Supp/ 1993;16:5-40.

21 Buajordet I, Ebbesen J, Erikssen J, et al. Fatal adverse drug events: the paradox of drug treatment. J Intern Med 2001;250:327-41.

22 Ambrosetti M, Ageno W, Spanevello A, et al. Prevalence and prevention of venous thromboembolism in patients with acute exacerbations of COPD. Thromb Res 2003:112:203-7.

23 Rizkallah J, Man SF, Sin DD. Prevalence of pulmonary embolism in acute exacerbations of COPD: a systematic review and metaanalysis. Chest 2009:135:786-93.

24 Thygesen $\mathrm{K}$, Alpert JS, White HD, et al. Universal definition of myocardial infarction. Circulation 2007:116:2634-53.

25 Brekke PH, Omland T, Holmedal SH, et al. Determinants of cardiac troponin elevation in COPD exacerbation-a cross-sectional study. BMC Pulm Med 2009;9:35.

26 Hoiseth $A D$, Omland $T$, Hagve $T A$, et al. Determinants of high-sensitivity cardiac troponin T during acute exacerbation of chronic obstructive pulmonary disease: a prospective cohort study. BMC Pulm Med 2012;12:22.

27 Vernooy JH, Kucukaycan M, Jacobs JA, et al. Local and systemic inflammation in patients with chronic obstructive pulmonary disease: soluble tumor necrosis factor receptors are increased in sputum. Am J Respir Crit Care Med 2002;166:1218-24.

28 Bathoorn E, Liesker JJ, Postma DS, et al. Change in inflammation in out-patient COPD patients from stable phase to a subsequent exacerbation. Int I Chron Obstruct Pulmon Dis 2009;4:101-9.

29 Hurst JR, Perera WR, Wilkinson TM, et al. Systemic and upper and lower airway inflammation at exacerbation of chronic obstructive pulmonary disease. Am J Respir Crit Care Med 2006:173:71-8. 